Cronfa - Swansea University Open Access Repository

This is an author produced version of a paper published in :

Southern Economic Journal

Cronfa URL for this paper:

http://cronfa.swan.ac.uk/Record/cronfa21493

\title{
Paper:
}

James, J. \& Lawler, P. (2015). Heterogeneous private sector information, central bank disclosure, and stabilization policy. Southern Economic Journal, 82(2), 620-634.

http://dx.doi.org/10.1002/soej.12028

This article is brought to you by Swansea University. Any person downloading material is agreeing to abide by the terms of the repository licence. Authors are personally responsible for adhering to publisher restrictions or conditions. When uploading content they are required to comply with their publisher agreement and the SHERPA RoMEO database to judge whether or not it is copyright safe to add this version of the paper to this repository. http://www.swansea.ac.uk/iss/researchsupport/cronfa-support/ 


\section{Heterogeneous Private Sector Information, Central Bank Disclosure and Stabilization Policy}

Jonathan G. James ${ }^{\dagger}$ and Phillip Lawler*

$\dagger \quad$ College of Business and Economics, Swansea University, Singleton Park, Swansea SA2 8PP, United Kingdom; E-mail: jonathan.g.james@swansea.ac.uk; corresponding author.

* College of Business and Economics, Swansea University, Singleton Park, Swansea SA2 8PP, United Kingdom; E-mail: p.lawler@swansea.ac.uk

Running Head: Heterogeneous Information, Disclosure and Stabilization

JEL Classification: D82; E52; E58.

Acknowledgements: The authors express thanks to an anonymous referee for helpful comments and suggestions. Generous financial support for the research contained in this paper from the Royal Economic Society (James) and the Leverhulme Trust (Lawler) is gratefully acknowledged. 
Abstract: Conventional wisdom has it that a central bank which employs an informational advantage to undertake active policy intervention can do as well, at least so far as real outcomes are concerned, by making its information publicly available and abstaining from stabilization. This notion is examined using a framework incorporating heterogeneous private sector information concerning aggregate demand shocks. An activist regime, in which the central bank exploits its own information to engage in stabilization, is found to be unambiguously superior to a non-interventionist regime, where the central bank maintains a constant setting of policy but publicly discloses its own information. 
“... if superior information is the basis for potential stabilization, there is a much easier way for the government to accomplish that stabilization than following a complex policy rule: it can simply announce the information that the public does not have." David Romer (2011, p.300)

\section{Introduction}

The advent of the rational expectations hypothesis posed a fundamental challenge to the belief that, by engaging in active stabilization policy, governments could improve macroeconomic outcomes relative to a laissez-faire approach, in which the (alleged) self-stabilizing properties of the economy are relied upon to return the economy to equilibrium following any exogenous shock. Although it was recognized that an information advantage on the part of the monetary authority might possibly provide the foundation for beneficial policy intervention (Barro, 1976), equally it was argued that essentially the same result could be achieved by the monetary authority making its own information publicly available. The conventional wisdom on this issue is well summarized by the above quotation from Romer (2011). Subsequently, any underpinnings for an interventionist stance on macroeconomic policy have been viewed as reliant on the presence of nominal rigidities which imply that wages and/or prices cannot react as quickly to information as monetary policy is able (important early contributions in this vein include Fischer, 1977; Taylor 1979, 1980).

The intention of the present paper is to re-examine the question of whether superior information might, in itself, provide the basis for the advocacy of activist monetary policy. The motive for revisiting this issue stems from the developing body of literature whose focus lies on the equilibrium and welfare properties of economies 
characterized by dispersed private information: see, for example, Morris and Shin (2002), Hellwig (2005), Angeletos and Pavan (2007a, 2007b), Cornand and Heinemann (2008), and James and Lawler (2011, 2012a,b). A finding emphasized by this literature is the possibility that, in the presence of heterogeneous information, strategic and external effects might lead to departures from efficiency. This is the case in the model employed in what follows, where such departures play a central role.

The framework described below, incorporating monopolistically-competitive pricesetting firms, derives from Woodford (2002, 2003), Adam (2007), and Roca (2010), with variants of it also applied in recent work by, for example, Baeriswyl and Cornand $(2010,2011)$ and Hahn (2014). Its key feature is that the pricing decisions of individual firms are associated with a strategic complementarity. In the presence of heterogeneous firm-specific information on the realized value of any shock, the strategic complementarity implies that the equilibrium price response to any public information released by the central bank is inefficient from the viewpoint of social welfare. The principal results of the paper then derive from this latter feature.

The focus of the study is the following issue: does an interventionist approach to monetary policy, directed at maximizing expected welfare, lead to outcomes superior in terms of the expected welfare criterion to those attained by passive policy? In addressing this question, activist policy is represented by a state-contingent rule which responds to the central bank's estimate of aggregate disturbances; the passive policy regime, on the other hand, is characterized by a fixed monetary rule combined with full public disclosure of the central bank's information. The logic underlying the view that disclosure by the monetary authority of its own information to the private sector can substitute for policy activism suggests that the two regimes are equivalent, as least 
insofar as their implications for real macroeconomic outcomes are concerned.

However, the key finding of the current study is that, despite the absence of price rigidities, activist policy achieves an unambiguously superior welfare outcome to that associated with the passive regime. We also demonstrate that this conclusion is robust to alternative informational assumptions to those that characterize the basic model.

The question which provides the main focus of the present study is, of course, closely related to that concerning the desirability, or otherwise, of central bank transparency. ${ }^{2}$ However, the two issues are distinct in a fundamental respect. Specifically, the modern debate regarding central bank transparency does not, in itself, reflect any difference of view regarding the need for active policy intervention. Rather, the interest lies in whether the efficacy of stabilization policy is improved or impaired by public disclosure of the central bank's information. On the other hand, the position represented in the quotations taken from Barro (1976) and Romer (2011) asserts definitively that central bank communication can serve as a substitute for policy activism and, in this regard, carries with it particularly strong implications for the appropriate conduct of policy. Notwithstanding this important distinction, our study does briefly consider the connection between the two issues in the context of the present model.

The remainder of the paper is structured as follows. Section 2 outlines the model and describes the informational assumptions which characterize the alternative policy regimes. Section 3 then identifies and compares the equilibrium outcomes under the two regimes and establishes the central result of the paper. Our findings are interpreted and related to those of other work concerned with the interaction between 
public and private information in Section 4. Section 5 then considers alternative information scenarios, before some brief concluding remarks are offered in Section 6 .

\section{The Model}

Underlying the component relationships of the model is a simple micro-founded general equilibrium framework developed in previous contributions by Woodford (2002, 2003), Adam (2007), and Roca (2010). In essence, the private sector comprises a representative household and a continuum of monopolistically-competitive firms, uniformly distributed over the unit interval. Household utility is defined over leisure and consumption, the latter described by a Dixit-Stiglitz CES aggregator over the varieties of differentiated goods.

Monetary policy is conducted by a central bank whose aim is to maximize household welfare, where the latter can be represented (as a second-order approximation) by:

$$
W=-\left[\int_{0}^{1}\left(p_{i}-p\right)^{2} d i+\lambda y^{2}\right]
$$

where $y$ represents the output gap, i.e. the deviation of actual output from its 'natural' level, ${ }^{3} p_{i}$ is the price set by firm $i$, and $p\left(\equiv \int_{0}^{1} p_{i} d i\right)$ identifies the average price across all firms (i.e. the price level). Thus, realized welfare for the household is dependent both on the degree of price dispersion and on the size of the output gap. The coefficient $\lambda$, which determines the relative significance of the two arguments of the welfare function, is a structural parameter whose value is dependent on the 
household's degree of risk aversion and the elasticity of substitution between goods in consumption, $\theta(>1)$.

Consideration of the profit maximization problem of the individual firm identifies its optimal price, $p_{i}$, to be a linear combination of the expected price level and output gap:

$$
p_{i}=E_{i}(p+\beta y)
$$

where $E_{i}$ represents firm $i$ 's expectation, conditional on the information available to it. With prices assumed to be perfectly flexible, each firm's actual price is always at its perceived optimal value. The responsiveness of $p_{i}$ to the expected output gap is determined by the parameter $\beta$ which identifies the degree of strategic complementarity between prices. In principle, it is possible for prices to be strategic substitutes but our analysis focuses on the realistic case of strategic complementarity, associated with $\beta \in(0,1){ }^{4}$ Important for the evaluation of welfare outcomes is the fact that the coefficients $\beta$ and $\lambda$ are related by $\lambda=\beta / \theta$, implying $\lambda<\beta$.

The setting of the central bank's policy instrument, $g$, determines the economy's level of aggregate nominal demand, $n$, subject to the realization of a stochastic aggregate demand shock, $\phi$, assumed to be drawn from a uniform distribution over the real line. ${ }^{5}$ Shocks to aggregate demand can be taken as representative of a broader class of shock which leave the relationship between the full information equilibrium and the associated socially optimal outcome unchanged. For shocks of this nature, which include those to production technology and to household preferences, the inefficiency referred to in the Introduction, and which underlies the key findings of this paper, 
derives from the presence of uncertainty: under complete information equilibrium outcomes would be efficient. An alternative category of shock, specifically any affecting the degree of monopoly power in goods and/or labor markets, gives rise to departures from efficiency even under complete information. Such shocks are invariably associated with fluctuations in the gap between full information equilibrium and socially optimal outcomes. Although these fluctuations introduce additional considerations relevant to the conduct of stabilization policy, they do not affect the conclusions drawn in the analysis which follows.

The relationship between aggregate nominal demand, the setting of the central bank's policy instrument and the aggregate demand shock can be expressed as $n(\equiv p+y)=g+\phi$. On rearrangement this becomes:

$$
y=g-p+\phi
$$

It follows from (3) that equation (1) can be rewritten as:

$$
W=-\left[\int_{0}^{1}\left(p_{i}-p\right)^{2} d i+\lambda(p-g-\phi)^{2}\right]
$$

while firm $i$ 's pricing equation (2) can now be expressed as:

$$
p_{i}=E_{i}[(1-\beta) p+\beta(g+\phi)]
$$

We note that in the current flexible price framework, price dispersion, as represented by the first term on the right hand side of $\left(1^{\prime}\right)$, arises only as a result of the heterogeneity of private information and the associated firm-specific expectational errors. ${ }^{6}$ On the other hand, any departure of the price level from the value which 
would eliminate the output gap, as captured by the second term of $\left(1^{\prime}\right)$, reflects expectational errors which are common across firms: such errors are associated with volatility in output. The presence within the model of heterogeneous information gives rise to a trade-off between (price) dispersion and (output) volatility, with the differing implications of the alternative policy regimes for the equilibrium trade-off central to this study's conclusions.

We now turn to the informational assumptions which characterize our analysis. Prices are set before the realized value of the aggregate demand shock is known. However, prior to making its pricing decision, firm $i$ observes an idiosyncratic noisy signal of $\phi$ : each firm's signal is private information in the sense that it cannot be observed by any other firm. We denote the signal received by firm $i$ by $\eta_{i}$, where:

$$
\eta_{i}=\phi+\xi_{i}
$$

The noise term, $\xi_{i} \sim N\left(0, \sigma_{\xi}^{2}\right)$, is assumed independent of $\phi$ with $E\left(\xi_{i} \xi_{j}\right)=0$ for $j \neq i$, while $\int_{0}^{1} \xi_{i} d i=0$.

Before setting its price, in addition to its private signal each firm may, at the discretion of the central bank, also have access to a common public signal. The central bank, like firms, does not know the true value of the aggregate demand shock before choosing any action of its own, but rather observes its own noisy signal, $\delta$, of $\phi$ 's realization, where: 


$$
\delta=\phi+\varepsilon
$$

The error term, $\varepsilon \sim N\left(0, \sigma_{\varepsilon}^{2}\right)$, is independent of $\phi$ and uncorrelated with the corresponding private sector noise terms, i.e. $E\left(\varepsilon \xi_{i}\right)=0, \forall i$. Whether or not the central bank reveals its own information to the private sector is central to the distinction between the alternative policy regimes discussed and analyzed in the next section.

\section{Equilibrium and Macroeconomic Stability under the Alternative Policy}

\section{Regimes}

Our interest in this section lies in determining whether the behavior of the real economy is independent of whether the central bank employs its own information to engage in active stabilization or, alternatively, simply reveals this information to the private sector and itself abstains from policy intervention. In examining this issue, we first identify the nature of the equilibrium associated with the activist regime, then consider macroeconomic outcomes when monetary policy is passive but the central bank announces its own information publicly.

\section{Policy activism with undisclosed central bank information}

The activist regime is characterized in terms of commitment to a policy rule which determines the setting of policy as a function of the central bank's current information. In this section we assume that neither the central bank nor any firm is able to observe the chosen action of any other agent prior to making its own decision. 
Thus firm $i$ cannot observe $g$ or any $p_{j}(j \neq i)$ prior to setting $p_{i}$, while the central bank cannot observe any $p_{i}$ before implementing its choice of $g$. It follows that the central bank's sole source of information regarding the realized value of $\phi$ is the signal, $\delta$ that it observes. Given the linear-quadratic structure of the model, optimal policy can be represented as a linear decision rule of the form:

$$
g=\rho \delta
$$

As well as the central bank's stance on policy intervention, also crucial to the distinction between the two regimes is the information on which private sector actions are based. Under the activist policy regime, the central bank's own signal is not part of any firm's information set. Hence pricing decisions are conditioned only on each firm's private signal. Noting that $E\left(\delta \mid \eta_{i}\right)=E\left(\phi \mid \eta_{i}\right)=\eta_{i}$, and using the policy rule described by (6), firm $i$ 's price can be expressed, from (2'), as:

$$
p_{i}=(1-\beta) E\left(p \mid \eta_{i}\right)+\beta(1+\rho) \eta_{i}
$$

To identify the equilibrium outcome of these pricing decisions, we use the method of undetermined coefficients, assuming a price response function of the form:

$$
p_{i}=\kappa_{0}+\kappa_{1} \eta_{i}
$$


Noting the property $\int_{i=0}^{1} \xi_{i} d i=0$, it follows that $p=\kappa_{0}+\kappa_{1} \phi$. Substituting this

expression for $p$ into (7), we find:

$$
p_{i}=(1-\beta) \kappa_{0}+\left[\beta(1+\rho)+(1-\beta) \kappa_{1}\right] \eta_{i}
$$

Equating coefficients across (8) and (9) yields:

$$
\kappa_{0}=0 \quad \kappa_{1}=1+\rho
$$

It follows that firm $i$ 's price and the price level are given respectively by:

$$
p_{i}=(1+\rho) \eta_{i} \quad(10 \mathrm{a}) ; \quad \quad p=(1+\rho) \phi
$$

Equation (10) implies that the departure of firm $i$ 's price from the economy-wide average price is given by:

$$
p_{i}-p=(1+\rho) \xi_{i}
$$

while using (6) and (10b) together with (3) allows the equilibrium output gap to be identified:

$$
y=\rho \varepsilon
$$


Equilibrium (unconditional) expected welfare can now be found by substituting equations (11) and (12) into (1) and applying the expectations operator:

$$
E(W)=-\left[(1+\rho)^{2} \sigma_{\xi}^{2}+\lambda \rho^{2} \sigma_{\varepsilon}^{2}\right]
$$

Minimizing the above expression over $\rho$ allows us to identify the optimal value, $\rho^{*}$, of the rule parameter:

$$
\rho^{*}=-\frac{\sigma_{\xi}{ }^{2}}{\sigma_{\xi}^{2}+\lambda \sigma_{\varepsilon}^{2}}
$$

Note that, outside the limiting cases of perfect central bank information $\left(\sigma_{\varepsilon}^{2}=0\right)$ and a completely uninformed private sector $\left(\sigma_{\xi}^{2} \rightarrow \infty\right),-1<\rho^{*}<0$. Hence, the optimal rule requires that policy only partially offset the central bank's expectation of the shock (i.e. $E(\phi \mid \delta)=\delta$ ). To explain this feature of optimal policy, we note that if monetary policy were designed to fully offset the central bank's estimate of $\phi$, the expectation of each firm would be that the setting of the monetary instrument would fully neutralize the impact of the shock (since, for $\rho=-1$, $\left.E_{i}(\phi+\rho \delta)=E\left(\phi+\rho \delta \mid \eta_{i}\right)=0\right)$. Consequently, prices would not respond at all to firms' private information. Although this would lead to the complete elimination of price dispersion, the associated welfare gain would be outweighed by the effect of the accompanying increase in output volatility, reflecting the non-utilization of the information content of firms' private signals. $^{8}$ 
Now substituting the expression for $\rho^{*}$ into (13), and using $E\left(\left.W^{A}\right|_{\rho=\rho^{*}}\right)$ to represent equilibrium expected welfare under activism when policy is set optimally:

$$
E\left(\left.W^{A}\right|_{\rho=\rho^{*}}\right)=-\frac{\lambda \sigma_{\xi}{ }^{2} \sigma_{\varepsilon}^{2}}{\sigma_{\xi}^{2}+\lambda \sigma_{\varepsilon}^{2}}
$$

Passive monetary policy with central bank announcements

We now turn to consider equilibrium outcomes when the central bank refrains from adjusting its setting of monetary policy in response to its own information, but discloses the observed value of its signal to the private sector. From the properties of $\phi, \delta$ and $\eta_{i}$, the optimal predictor of $\phi$ conditional on $\eta_{i}$ and $\delta$ is:

$$
E_{i} \phi=E\left(\phi \mid \eta_{i}, \delta\right)=\gamma \eta_{i}+(1-\gamma) \delta, \quad \gamma \equiv \sigma_{\varepsilon}^{2} /\left(\sigma_{\varepsilon}^{2}+\sigma_{\xi}^{2}\right)
$$

For comparability with the activist regime, we normalize the fixed setting of the central bank's instrument to zero (i.e. $g \equiv 0$ ) so that the mean level of nominal income is identical under the alternative policy regimes. Equation $\left(2^{\prime}\right)$ then implies:

$$
p_{i}=(1-\beta) E\left(p \mid \eta_{i}, \delta\right)+\beta\left[\gamma \eta_{i}+(1-\gamma) \delta\right]
$$

We again determine equilibrium by employing the method of undetermined coefficients, positing that firm $i$ 's price is now set according to: 


$$
p_{i}=k_{0}+k_{1} \eta_{i}+k_{2} \delta
$$

which implies that the price level is described by $p=k_{0}+k_{1} \phi+k_{2} \delta$. Substituting the expected value (conditional on $\eta_{i}$ and $\delta$ ) of this expression into (17):

$$
p_{i}=(1-\beta) k_{0}+\gamma\left[\beta+(1-\beta) k_{1}\right] \eta_{i}+\left\{\beta(1-\gamma)+(1-\beta)\left[(1-\gamma) k_{1}+k_{2}\right]\right\} \delta
$$

Equating coefficients across (18) and (19) and using the definition of $\gamma$ we find:

$$
k_{0}=0 \quad k_{1}=\frac{\beta \sigma_{\varepsilon}^{2}}{\sigma_{\xi}^{2}+\beta \sigma_{\varepsilon}^{2}} \quad k_{2}=\frac{\sigma_{\xi}^{2}}{\sigma_{\xi}^{2}+\beta \sigma_{\varepsilon}^{2}}
$$

Substituting these expressions into (18), then aggregating over firms, identifies the equilibrium values of $p_{i}$ and $p$ :

$$
p_{i}=\frac{\beta \sigma_{\varepsilon}^{2} \eta_{i}+\sigma_{\xi}^{2} \delta}{\sigma_{\xi}^{2}+\beta \sigma_{\varepsilon}^{2}} \quad(20 \mathrm{a}) ; \quad p=\frac{\beta \sigma_{\varepsilon}^{2} \phi+\sigma_{\xi}^{2} \delta}{\sigma_{\xi}^{2}+\beta \sigma_{\varepsilon}^{2}}
$$

Hence the deviation of $p_{i}$ from $p$ is:

$$
p_{i}-p=\frac{\beta \sigma_{\varepsilon}^{2} \xi_{i}}{\sigma_{\xi}^{2}+\beta \sigma_{\varepsilon}^{2}}
$$

while substituting (20b) into (3) yields: 


$$
y=-\frac{\sigma_{\xi}^{2} \varepsilon}{\sigma_{\xi}^{2}+\beta \sigma_{\varepsilon}^{2}}
$$

Equilibrium expected welfare in the case of passive policy, which we denote by $E\left(W^{P}\right)$, is then found by substituting equations (21) and (22) into (1) :

$$
E\left(W^{P}\right)=-\frac{\left(\lambda \sigma_{\xi}^{2}+\beta^{2} \sigma_{\varepsilon}^{2}\right) \sigma_{\xi}^{2} \sigma_{\varepsilon}^{2}}{\left(\sigma_{\xi}^{2}+\beta \sigma_{\varepsilon}^{2}\right)^{2}}
$$

Inspection of equations (15) and (23) reveals that, apart from the special cases in which one or other of the central bank or the private sector observes either a fully informative or a completely uninformative signal, the two policy regimes are characterized by different welfare outcomes. Noting that, as previously identified, $\lambda<\beta$, direct comparison of equations (15) and (23) allows us to formulate the following proposition:

PROPOSITION 1: Passive monetary policy with public disclosure of the central bank's signal is associated with an unambiguously lower value of expected welfare than the activist regime, i.e. $E\left(W^{P}\right)<E\left(\left.W^{A}\right|_{\rho=\rho^{*}}\right)$

The finding summarized by Proposition 1, which represents the principal conclusion of the paper, stands in clear contrast to the conventional wisdom, as exemplified in the quotation from Romer (2011) reproduced earlier. In the next section we turn to consider the economic logic which underlies this result. 


\section{Discussion}

The key to the comparative welfare properties of the alternative policy regimes lies in the strategic complementarity which underlies firms' pricing decisions. As noted in the Introduction, in the presence of heterogeneous information, strategic complementarity (or substitutability) can lead agents to respond to that information inefficiently. Central to the nature of this inefficiency is the distinction drawn by Angeletos and Pavan (2007b) between the 'equilibrium degree of coordination' and the 'socially optimal degree of coordination'. The former identifies the private benefit that individuals place on aligning their own actions with those of others, while the latter relates to the social benefit of such alignment. In the present context, with $\beta>\lambda$, the equilibrium degree of coordination, $1-\beta$, lies below the socially optimal degree of coordination, $1-\lambda$. The relevance of this feature of the model for the characteristics of equilibrium under passive monetary policy can be demonstrated by determining the collectively-optimal individual price responses to private and public signals within this regime.

We again specify the individual firm's price to be a linear function of $\eta_{i}$ and $\delta$ :

$$
p_{i}=\widetilde{k}_{0}+\widetilde{k}_{1} \eta_{i}+\widetilde{k}_{2} \delta
$$

implying the average price is given by $p=\widetilde{k}_{0}+\widetilde{k}_{1} \phi+\widetilde{k}_{2} \delta$. The consequent expression for the deviation of firm $i$ 's price from the price level is simply $p_{i}-p=\widetilde{k}_{1} \xi_{i}$, while

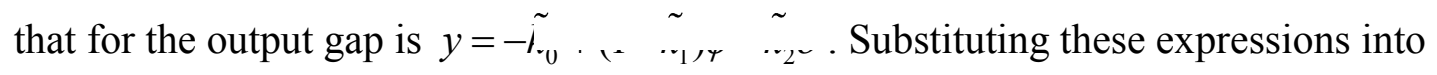
(1), the first order conditions for $\widetilde{k}_{0}, \widetilde{k}_{1}$ and $\widetilde{k}_{2}$ then yield: 


$$
\tilde{k}_{0}=0 \quad \tilde{k}_{1}=\frac{\lambda \sigma_{\varepsilon}^{2}}{\sigma_{\xi}^{2}+\lambda \sigma_{\varepsilon}^{2}} \quad \tilde{i}_{2} \quad \sigma_{\xi}^{2}+\overline{\lambda \sigma_{\varepsilon}^{2}}
$$

It is straightforward to confirm that $\widetilde{k}_{1}<k_{1}$ and, hence, $k_{2}<\widetilde{k}_{2}$. Therefore, in the context of passive monetary policy with public disclosure of the central bank's information, each firm's price response places excessive weight, relative to the efficient level, on private information. This characteristic derives from the fact, identified above, that the equilibrium degree of coordination is less than the efficient degree of coordination: that is, the incentives which face individual firms to align their prices with those set by other firms understate the collective benefit of alignment.

The values of $p_{i}$ and $p$ associated with efficient responses to the observed signals can be identified as:

$$
p_{i}=\frac{\lambda \sigma_{\varepsilon}^{2} \eta_{i}+\sigma_{\xi}^{2} \delta}{\sigma_{\xi}^{2}+\lambda \sigma_{\varepsilon}^{2}} \quad(25 \mathrm{a}) ; \quad p=\frac{\lambda \sigma_{\varepsilon}^{2} \phi+\sigma_{\xi}^{2} \delta}{\sigma_{\xi}^{2}+\lambda \sigma_{\varepsilon}^{2}}
$$

with the departure of $p_{i}$ from $p$ in this instance described by:

$$
p_{i}-p=\frac{\lambda \sigma_{\varepsilon}^{2} \xi_{i}}{\sigma_{\xi}^{2}+\lambda \sigma_{\varepsilon}^{2}}
$$


and the output gap equal to:

$$
y=-\frac{\sigma_{\xi}^{2} \varepsilon}{\sigma_{\xi}^{2}+\lambda \sigma_{\varepsilon}^{2}}
$$

Comparison of equations (21) and (22) with (26) and (27) establishes that, for given signal observations, equilibrium price-setting leads to larger departures of the individual firm price from the average value, but a smaller output gap, than is socially optimal. Alternatively put, the equilibrium trade-off between dispersion and volatility under passive policy diverges from the socially-optimal trade-off. This feature reflects the overweighting of private information in equilibrium price responses to the observed signals, which acts to exacerbate the effect of idiosyncratic errors on price dispersion. The effect of this on expected welfare is mitigated somewhat by the reduction in output gap volatility, as the underweighting of the public signal implies a lessened degree of commonality in pricing behavior. However, the net effect is an unambiguous fall in expected welfare below the value associated with sociallyefficient price-setting.

Now substituting (26) and (27) into (1) to determine the resulting value of expected welfare, $E\left(\widetilde{W}^{P}\right)$, when policy is passive but prices respond to information in an efficient manner:

$$
E\left(\widetilde{W}^{P}\right)=-\frac{\lambda \sigma_{\xi}{ }^{2} \sigma_{\varepsilon}^{2}}{\sigma_{\xi}^{2}+\lambda \sigma_{\varepsilon}^{2}}
$$


Given the inefficiency which characterizes equilibrium price-setting under passive policy, it is evident that $E\left(W^{P}\right)<E\left(\widetilde{W}^{P}\right)$, as is straightforward to confirm by comparison of (23) with (28). However, comparing (15) with (28), it is apparent that expected welfare under the activist regime is identical to that which would be achieved under passive monetary policy if prices were to respond efficiently to the observed private and public signals. In this sense, active policy intervention enables the attainment of the first best outcome. ${ }^{9}$

The ability of the activist regime to ensure that all available information is exploited efficiently can be explained as follows. In the presence of the strategic complementarity discussed above, the equilibrium price-setting strategy of firm $i$ defines $p_{i}$ as a weighted average of the firm's expectation of the 'fundamentals' (i.e. the net impact of the shock, $\phi$, and monetary policy) and its expectation of the average price, ${ }^{10}$ as represented by $\left(2^{\prime}\right)$. If the central bank does not disclose its own information regarding $\phi$, then firm $i$ 's best estimate of $p$ is simply its own price, i.e. $E_{i}(p)=p_{i}$. Thus $p_{i}=E_{i}(g+\phi)$, with the direct effect of the strategic complementarity on price setting eliminated. Moreover, since each firm's expectations are formed solely on the basis of its own private signal, $E_{i}(\delta)=E_{i}(\phi)=\eta_{i}$ : hence $p_{i}=(1+\rho) \eta_{i}$. For $\rho \in(-1,0)$, it is clear that the more responsive policy is to the central bank's signal the less responsive prices will be to firm-specific signals. The value of the rule parameter therefore determines the relative weights placed on the different sources of information in the joint responses of monetary policy and prices to shocks. The optimal value of $\rho$ ensures that these weights reflect both the relative accuracy of the two signals and the social value of 
price coordination. Hence it leads, in contrast to the passive policy regime, to the efficient use of available information.

The principles outlined in the preceding discussion are also central to the conclusions drawn in James and Lawler (2011, 2012a,b). In each of these studies, abstract models incorporating heterogeneous information are applied to examine the social welfare consequences of public disclosure of information that is otherwise private to the policymaker in the presence of optimal stabilization policy. Their concern thus lies with the issue that provides the focus of the modern debate relating to central bank transparency. As noted in the Introduction, this issue is distinct from the question considered in the present contribution, that is whether public dissemination of the central bank's information can eliminate the need for active policy intervention. Nonetheless, the two issues are evidently related. In order to identify the precise nature of the connection between them, we use the current framework to briefly consider the implications of central bank disclosure of its signal, $\delta$, in the context of the activist regime.

In this instance, the central bank, besides using the observation of its signal to determine the setting of its policy instrument, as described by (6), also publicly announces the value of $\delta$. The latter thus becomes part of the private sector's information set, as in the passive policy regime examined in Section 3. Importantly, in addition to modifying each firm's estimate of $\phi$ compared to when only its own signal is observed, this also provides precise knowledge of the central bank's instrument setting. 
In order to determine equilibrium in the present context, the same steps are followed as in Section 3's consideration of the activist regime, but now recognizing that firm $i$ sets its price conditional not only on $\eta_{i}$, but also on $\delta$. Denoting firm $i$ 's equilibrium price under policy activism but with disclosure of the central bank's signal by $p_{i}^{A(D)}$, and under passive policy, as described by (20a), by $p_{i}^{P}$, we find that $p_{i}^{A(D)}=p_{i}^{P}+\rho \delta$. It follows immediately from the implied value of $p, p^{A(D)}$, that public disclosure of $\delta$ implies that price dispersion in the activist regime becomes identical to that under passive policy. This isomorphism extends to the behavior of aggregate output, as is evident from substituting (6), together with the value for $p^{A(D)}$ , into (3). Hence, in announcing $\delta$ publicly, the central bank renders policy completely ineffective and engenders an identical welfare outcome to that attained when policy is passive. It follows that Proposition 1 indirectly identifies central bank transparency to be undesirable in the context of activist policy. ${ }^{11}$

The logic underlying this finding is discussed in the aforementioned papers by James and Lawler (2011, 2012a,b). In essence, due to its underestimation of the value of aligning its price with those of others, the individual firm regards socially-optimal policy intervention as sub-optimal from the viewpoint of its own objectives. In making its own signal common knowledge, the central bank provides each firm with the information that it needs to offset, by appropriate adjustment of its price, the perceived detrimental impact of policy on its profitability. Of course, with all firms acting in the same way, the economy is driven to an inferior equilibrium. ${ }^{12}$ 
We note that this conclusion appears, at first sight, to be at variance with those of Roca (2010) and Baeriswyl and Cornand (2011). Both papers consider the desirability of central bank transparency within essentially the same model as employed in the current study, with each finding that, for empirically plausible parameter values, transparency is beneficial to welfare. However, the difference with our own conclusions can be explained by the fact that both in Roca and in Baeriswyl and Cornand the price level is assumed to be a component of the policy authority's objectives. Given that it is the dispersion of prices around the average, rather than the absolute value of the latter, which is important for welfare, the formulation of policy in the two papers can be regarded as non-optimal from the viewpoint of the model's underlying welfare function, as described by (1). With policy conducted nonoptimally, it is clearly possible for public dissemination of the information on which this policy is based to improve welfare. ${ }^{13}$

\section{Alternative Information Scenarios}

We return to the main theme of the paper by considering two modifications to the informational assumptions which have characterized the analysis to this point, both of which relate to the potential sources of public information. The first recognizes the validity of Svensson's (2006, p.451) observation, in commenting on Morris and Shin (2002), that there is “... always some public information (available)..." regardless of central bank disclosure. Thus an additional public signal, outside the control of the central bank and independent of the policy regime, is introduced into the model. ${ }^{14}$ The second amends the assumed timing of moves which has characterized the analysis of 
the activist regime to this point, and allows each firm to observe (subject to an idiosyncratic error) the setting of policy before determining its price.

\section{All firms observe an additional common signal}

We now assume that under both policy regimes the central bank and the private sector each observes an imperfect public signal of the aggregate demand shock, $v=\phi+\psi$, where $\psi \sim N\left(0, \sigma_{\psi}^{2}\right)$ and is uncorrelated both with any $\xi_{i}$ and with $\varepsilon$. In considering the activist regime, the policy rule must incorporate a potential response to $v$ in addition to that associated with $\delta$. Hence the rule is now described by $g=\rho_{1} \delta+\rho_{2} v$. Applying the same solution steps to those employed in the analysis of Section 3, equilibrium price setting under policy activism is found to give rise to a departure of firm $i$ 's price from the average price as given by:

$$
p_{i}-p=\frac{\beta \sigma_{\psi}^{2}\left(1+\rho_{1}\right) \xi_{i}}{\left(\sigma_{\xi}^{2}+\beta \sigma_{\psi}^{2}\right)}
$$

while aggregate output is determined according to:

$$
y=-\frac{\sigma_{\xi}^{2}\left(1+\rho_{1}\right) \psi}{\left(\sigma_{\xi}^{2}+\beta \sigma_{\psi}^{2}\right)}+\rho_{1} \varepsilon
$$

We note that the policy rule parameter, $\rho_{2}$, relating to the common signal, $v$, is not present in these expressions. Reflecting the logic discussed in the preceding section, the socially-optimal policy response to $v$ is viewed by price-setters as sub-optimal 
from their individualistic perspectives. With common knowledge of $v$ rendering any systematic policy response to its observed value perfectly anticipatable, firms will adjust prices in such a way as to neutralize the potential impact on real outcomes of any policy reaction to the common public signal. It follows that $\rho_{2}$ can be set at any value, without any consequences for expected welfare.

To identify expected welfare under policy activism, (29) and (30) are substituted into equation (1), and the resulting expression maximized over $\rho_{1}$. The identified optimal value of the policy rule parameter, $\rho_{1}^{*}$, is then placed into the expressions for $p_{i}-p$ and $y$ before using (1) to evaluate expected welfare when policy is set optimally. We find:

$$
E\left(\left.W^{A}\right|_{\rho_{1}=\rho_{1}^{*}}\right)=-\frac{\sigma_{\xi}^{2} \sigma_{\psi}^{2} \sigma_{\varepsilon}^{2} \lambda\left(\beta^{2} \sigma_{\psi}^{2}+\lambda \sigma_{\xi}^{2}\right)}{\lambda \sigma_{\xi}^{4}\left(\sigma_{\psi}^{2}+\sigma_{\varepsilon}^{2}\right)+\beta^{2} \lambda \sigma_{\psi}^{4} \sigma_{\varepsilon}^{2}+\beta\left(\beta \sigma_{\psi}^{2}+2 \lambda \sigma_{\varepsilon}^{2}\right) \sigma_{\xi}^{2} \sigma_{\psi}^{2}}
$$

As might be expected, in the present scenario expected welfare under policy activism is generally higher than when there is no additional public signal (the exception being the extreme case which arises as $\sigma_{\psi}^{2} \rightarrow \infty$ and $v$ becomes completely uninformative: in this instance, equation (31) is identical to (15)). Although, as noted, any reaction of policy to observations of $v$ is superfluous to welfare, firms use the information embodied in this public signal to improve their estimates of both $\phi$ and $p$ and modify their prices accordingly. While this additional information is not used efficiently by price setters, as discussed in Section 4 any improvement in the quality of public information is welfare-improving within the current framework. 
Now considering equilibrium under passive policy, with the private sector directly observing $v$ as well as having the value of $\delta$ revealed to it by the central bank, it is straightforward to find, using the methodology previously applied in the context of this regime, the expressions describing $p_{i}-p$ and $y$. These are, respectively:

$$
\begin{aligned}
& p_{i}-p=\frac{\beta \sigma_{\psi}^{2} \sigma_{\varepsilon}^{2} \xi_{i}}{\sigma_{\xi}^{2}\left(\sigma_{\psi}^{2}+\sigma_{\varepsilon}^{2}\right)+\beta \sigma_{\psi}^{2} \sigma_{\varepsilon}^{2}} \\
& y=-\frac{\sigma_{\xi}^{2}\left(\sigma_{\psi}^{2} \varepsilon+\sigma_{\varepsilon}^{2} \psi\right)}{\sigma_{\xi}^{2}\left(\sigma_{\psi}^{2}+\sigma_{\varepsilon}^{2}\right)+\beta \sigma_{\psi}^{2} \sigma_{\varepsilon}^{2}}
\end{aligned}
$$

Using the above expressions in conjunction with equation (1), we identify expected welfare with passive monetary policy but the central bank's signal announced publicly:

$$
E\left(W^{P}\right)=-\frac{\sigma_{\xi}^{2} \sigma_{\psi}^{2} \sigma_{\varepsilon}^{2}\left[\beta^{2} \sigma_{\psi}^{2} \sigma_{\varepsilon}^{2}+\lambda \sigma_{\xi}^{2}\left(\sigma_{\psi}^{2}+\sigma_{\varepsilon}^{2}\right)\right]}{\left[\sigma_{\xi}^{2}\left(\sigma_{\psi}^{2}+\sigma_{\varepsilon}^{2}\right)+\beta \sigma_{\psi}^{2} \sigma_{e}^{2}\right]^{2}}
$$

Direct comparison of (34) with (31) indicates that $E\left(W^{P}\right)<E\left(\left.W^{A}\right|_{\rho_{1}=\rho_{1}^{*}}\right)$. Thus, the presence of an additional signal which is observed both by the central bank and the private sector does not modify our key finding that any information that is known only to the central bank should not be disclosed publicly, but rather should be reserved to provide the basis for active policy intervention. ${ }^{15}$ 


\section{Imperfect private sector observations of policy before prices are set}

Our analysis of the activist regime is now modified by assuming that all firms observe the setting of the central bank's instrument prior to determining their optimal price. However, these observations are assumed subject to firm-specific errors, with firm $i$ observing $\chi_{i}$, where $\chi_{i} \equiv g+\omega_{i}$. In this context, policy plays an (imperfect) signaling role ${ }^{16}$ and provides information additional to that embodied in the private $\eta_{i}$ signals, not only in respect of the setting of policy itself and the information on which this setting is based, but also regarding $\phi$. Recent papers which incorporate a signaling role for policy include Walsh (2007), Baeriswyl and Cornand (2010, 2011), and Hahn (2014).

Of course, under the passive policy regime the setting of the instrument is fixed, while the central bank's signal is disclosed directly to the private sector: consequently, expected welfare is described, as in the analysis of Section 3, by equation (23). In considering activist policy we report directly the expression for expected welfare: ${ }^{17}$

$$
\begin{aligned}
& E\left(W^{A}\right)= \\
& -\frac{\left[(1+\rho) \sigma_{\omega}^{2}+\beta \sigma_{\varepsilon}^{2} \rho^{2}\right]^{2} \sigma_{\xi}^{2}+\rho^{2}\left[(1+\rho) \sigma_{\xi}^{2}+\beta \sigma_{\varepsilon}^{2} \rho\right]^{2} \sigma_{\omega}^{2}+\lambda \rho^{2}\left(\rho \sigma_{\xi}^{2}-\sigma_{\omega}^{2}\right)^{2} \sigma_{\varepsilon}^{2}}{\left[\sigma_{\omega}^{2}+\left(\sigma_{\xi}^{2}+\beta \sigma_{\varepsilon}^{2}\right) \rho^{2}\right]^{2}}
\end{aligned}
$$

In the present case, a closed form solution for the optimal setting of the rule parameter is precluded by the complexity of the relationship between $E\left(W^{A}\right)$ and $\rho$. However, the following Proposition establishes a key result: 
PROPOSITION 2: Define $\hat{\rho} \equiv-\sigma_{\xi}^{2} /\left(\sigma_{\xi}^{2}+\beta \sigma_{\varepsilon}^{2}\right)$. Then, evaluating expected welfare for $\rho=\hat{\rho}$, we find $E\left(\left.W^{A}\right|_{\rho=\hat{\rho}}\right)=E\left(W^{P}\right)$. Moreover, the derivative of $E\left(W^{A}\right)$ with respect to $\rho$ evaluated at $\rho=\hat{\rho}$ is non-zero. It follows that with $\rho$ set optimally expected welfare resulting from activist policy is unambiguously higher than under the passive policy regime.

The result identified by Proposition 2 reflects the feature that, with the policy parameter set at $\hat{\rho}$, price-setters regard the central bank's policy response to its observed signal, $\delta$, to be optimal from their individual standpoints. As a consequence, the information contained in firms' noisy observations of $g$ does not affect pricing decisions. Significantly, with this non-optimal (from the perspective of social welfare) setting of $\rho$, i.e. $\hat{\rho}$, attaining the identical welfare outcome to that associated with the passive policy regime, it follows that the latter must be characterized by lower welfare than is socially-optimal policy intervention. ${ }^{18}$

\section{Concluding Comments}

The focus of this paper has been the question of whether public disclosure of the central bank's information concerning aggregate shocks can serve as a substitute for active stabilization policy. The preceding analysis clearly indicates that the answer to this question is in the negative. This is so despite the absence in our framework of any timing advantage for monetary policy, deriving from nominal rigidities which might prevent prices responding to new information as quickly as the central bank's policy setting is able. 
The crucial factor underlying this finding is, as discussed, the presence of heterogeneous private sector information which, in the presence of a strategic complementarity in price-setting, gives rise to a collectively-inefficient price response to central bank announcements. This inherent inefficiency is such as to imply that activist monetary policy, based on the central bank's information, attains an unambiguously superior macroeconomic outcome. Alternative information scenarios, in which the private sector has additional information and which, under the activist regime, allows an improved estimate by firms of the setting of policy, leave this unequivocal conclusion unaffected. Moreover, while the framework in which this study's findings were derived has a relatively basic form, it can be viewed as representative of a wider class of micro-founded macroeconomic models. Thus we would expect our principal results to remain robust to extensions of the analysis to more complex model structures. 


\section{References}

Adam, Klaus. 2007. Optimal monetary policy with imperfect common knowledge. Journal of Monetary Economics 54: 276-301.

Angeletos, George-Marios, and Alessandro Pavan. 2007a. Socially optimal coordination: Characterization and policy implications. Journal of the European Economic Association 5: 585-93.

Angeletos, George-Marios, and Alessandro Pavan. 2007b. Efficient use of information and social value of information. Econometrica 75: 1103-42.

Baeriswyl, Romain, and Camille Cornand. 2010. The signaling role of policy actions. Journal of Monetary Economics 57: 682-95.

Baeriswyl, Romain, and Camille Cornand. 2011. Transparency and monetary policy effectiveness. Annals of Economics and Statistics 103/104: 175-94.

Barro, Robert J. 1976. Rational expectations and the role of monetary policy. Journal of Monetary Economics 2: 1-32.

Blinder, Alan S., Michael Ehrmann, Marcel Fratzscher, Jakob De Haan, and DavidJan Jansen. 2008. Central bank communication and monetary policy: A survey of theory and evidence. Journal of Economic Literature 46: 910-45.

Cornand, Camille, and Frank Heinemann. 2008. Optimal degree of public information dissemination. Economic Journal 118: 718-42.

Fischer, Stanley. 1977. Long-term contracts, rational expectations and the optimal money supply rule. Journal of Political Economy 85: 191-205.

Geraats, Petra M. 2002. Central bank transparency. Economic Journal 112: F532-65. de Haan, Jakob, Sylvester C.W. Eijffinger, and Krzysztof Rybinski. 2007. Central bank transparency and central bank communication: Editorial introduction. European Journal of Political Economy 23, 1-8. 
Hahn, Volker. 2014. Transparency in monetary policy, signaling, and heterogeneous information. Macroeconomic Dynamics 18: 369-94.

Hellwig, Christian. 2005. Heterogeneous information and the benefits of public information disclosures. Unpublished paper, University of California Los Angeles. James, Jonathan G., and Phillip Lawler. 2011. Optimal policy intervention and the social value of information. American Economic Review 101: 1561-74.

James, Jonathan G., and Phillip Lawler. 2012a. Strategic complementarity, stabilization policy and the optimal degree of publicity. Journal of Money, Credit and Banking 44: 551-72.

James, Jonathan G., and Phillip Lawler. 2012b. Heterogeneous information quality, strategic complementarities and optimal policy design. Journal of Economic Behavior and Organization 83: 342-52.

Morris, Stephen, and Hyun Song Shin. 2002. Social value of public information. American Economic Review 92: 1521-34.

Roca, Mauro F. 2010. Transparency and monetary policy with imperfect common knowledge. IMF Working Paper WP/10/91.

Romer, David H. 2011. Advanced Macroeconomics. $4^{\text {th }}$ edition. New York: McGrawHill.

Sargent, Thomas, and Neil Wallace. 1975. 'Rational' expectations, the optimal monetary instrument, and the optimal money supply rule. Journal of Political Economy 83: 241-54.

Svensson, Lars E.O. 2006. Social value of public information: Morris and Shin (2002) is actually pro transparency not con. American Economic Review 96: 448-52. Taylor, John B. 1979. Staggered price setting in a macro model. American Economic Review 69: 108-13. 
Taylor, John B. 1980. Aggregate dynamics and staggered contracts. Journal of Political Economy 88: 1-23.

Walsh, Carl E. 2007. Optimal economic transparency. International Journal of Central Banking 3: 5-36.

Woodford, Michael. 2002. Inflation stabilization and welfare. Contributions to Macroeconomics 2: 1-53.

Woodford, Michael. 2003. Imperfect common knowledge and the effects of monetary policy. In Knowledge, Information, and Expectations in Modern Macroeconomics: In Honor of Edmund S. Phelps, edited by Phillipe Aghion, Roman Frydman, Joseph E. Stiglitz and Michael Woodford. Princeton, NJ: Princeton University Press, pp.25-58.

${ }^{1}$ Barro (1976, p.23), for example, asserts “... when the monetary authority has superior information about the economy, the provision of the information to the public is an alternative to an active stabilization policy", while Fischer (1977, p.194) concedes: "Superior information is ... a weak reed on which to base the argument for the effectiveness of monetary policy ...". Of course, the issue of whether the sharing of the monetary authorities' information with the private sector obviates the need for an activist policy stance is reflective of the wider debate on the (in)effectiveness of policy initiated by the classic paper of Sargent and Wallace (1975), and associated with the development of a vast literature during the late 1970s and early 1980s.

${ }^{2}$ The transparency literature is well surveyed in papers by Geraats (2002), de Haan et al. (2007) and Blinder et al. (2008).

${ }^{3}$ Within the model the natural output level (the output level which would prevail under full information) is constant. For convenience, we normalize its value at zero and, henceforth, use the terms 'output gap' and 'output' interchangeably. Note that, 
because of monopoly power in the goods market, the natural level of output diverges from the socially-optimal output level: this divergence represents a deadweight loss which is independent of the policy regime for the type of shock considered here and is therefore suppressed from the welfare function (1).

${ }^{4}$ Note that, for a given value of nominal aggregate demand, $n \equiv p+y$, equation (2) can be written as $p_{i}=E_{i}[(1-\beta) p+\beta n]$ with, for $\beta \in(0,1), p_{i}$ strictly increasing in $E_{i} p$

${ }^{5}$ Assuming a normally distributed shock (as, for example, in Adam, 2007) would not affect the conclusions reported below in any way.

${ }^{6}$ In models in which some prices are 'sticky' an additional source of price dispersion is introduced: see Woodford (2002) for the implications of different approaches to modeling price inertia.

${ }^{7}$ In the current setting, the optimal policy rule is unique only up to an additive constant: for convenience we set the value of this constant at zero.

${ }^{8}$ The principles underlying the optimal design of policy are discussed more fully in Section 4 .

9 'First best' in this context refers to the maximum attainable welfare level subject to the given degree of information dispersion.

${ }^{10}$ This reflects a general principle: see Angeletos and Pavan (2007b) p.1112.

${ }^{11}$ If the central bank is unable, for whatever reason, to undertake stabilization policy, then expected welfare is invariably higher when the central bank reveals its signal publicly, i.e. transparency is always welfare-improving under the passive policy regime. 
${ }^{12}$ In James and Lawler (2012b), transparency is not unambiguously inferior to nondisclosure. This finding reflects the assumption adopted in that paper that the precision of private signals is not uniform across the private sector, which implies that stabilization policy is unable to achieve the first best welfare outcome, even in the absence of transparency.

${ }^{13}$ We note that Baeriswyl and Cornand's (2010) analysis of transparency assumes policy to be directed at maximizing a welfare function equivalent to (1) and arrive at conclusions similar to those identified above.

${ }^{14}$ A specific example of such a signal arises if $\phi$ exhibits some persistence: see the online appendix to James and Lawler (2011) for discussion of this in the context of the abstract model of that paper.

${ }^{15}$ From this principle it follows that if the central bank had the capacity to prevent private sector access to $v$ but could adjust policy in response to its own observations of this signal, welfare under policy activism would be improved further.

${ }^{16}$ If the instrument setting could be observed perfectly by firms before prices were set, then policy would be rendered completely ineffective and the activist and passive policy regimes would be associated with identical welfare outcomes. We further note that the presence of transmission mechanism shocks, observed by the central bank but not the private sector, would imply that the central bank's signal could not be inferred precisely by firms even from exact observations of policy. This reintroduces policy effectiveness under the activist regime even in the absence of observational errors regarding the policy setting (see James and Lawler, 2012a).

${ }^{17}$ The deviation of $p_{i}$ from $p$ is: $p_{i}-p=\left\{\left[(1+\rho) \sigma_{\omega}^{2}+\beta \sigma_{\varepsilon}^{2} \rho^{2}\right] \xi_{i}+\rho\left[(1+\rho) \sigma_{\xi}^{2}+\beta \sigma_{\varepsilon}^{2} \rho\right] \omega_{i}\right\}\left[\sigma_{\omega}^{2}+\left(\sigma_{\xi}^{2}+\beta \sigma_{\varepsilon}^{2}\right) \rho^{2}\right]^{-1}$, 
while output is described by: $y=\rho\left(\sigma_{\omega}^{2}-\rho \sigma_{\xi}^{2}\right) \varepsilon\left[\sigma_{\omega}^{2}+\left(\sigma_{\xi}^{2}+\beta \sigma_{\varepsilon}^{2}\right) \rho^{2}\right]^{-1}$.

${ }^{18}$ The logic underlying this argument is perfectly general and is used to establish the non-optimality of complete transparency by the policymaker in the context of the abstract model employed in James and Lawler (2012a). 